\title{
Sensory taste preferences and taste sensitivity and the association of unhealthy food patterns with overweight and obesity in primary school children in Europe-a synthesis of data from the IDEFICS study
}

Wolfgang Ahrens on behalf of the IDEFICS consortium

\begin{abstract}
Background: Increased preference for fat and sugar or reduced taste sensitivity may play a role in overweight and obesity development, but sensory perceptions are probably influenced already during childhood by food cultures and common dietary habits. We summarise the main findings of a large-scale epidemiological study conducted in Italy, Estonia, Cyprus, Belgium, Sweden, Germany, Hungary and Spain. We measured the taste preferences and the taste thresholds in 1,839 children aged 6 to 9 years and investigated factors that might influence the observed preferences as well as their association with weight status.

Findings: Country of residence was the strongest factor related to preferences for sweet, salty, bitter and umami. Taste preferences also differed by age. Regardless of the country of residence and other covariates, overweight and obesity were positively associated with the preference for fat-enriched crackers and sugar-sweetened apple juice.

Conclusions: We conclude that culture and age are important determinants of taste preferences in pre-adolescent children. The cross-sectional data show that objectively measured taste preferences are associated with the weight status of primary school children across varying food cultures. We hypothesise that this association is mediated by an unfavourable food choice as a food pattern characterised by sweet and fatty foods is associated with excess weight gain in these children.
\end{abstract}

Keywords: Cross-sectional study, Epidemiology, Food culture, Measurement of taste qualities, Overweight and obesity, Sensory taste perception, Bitter taste, Salty taste, Sweet taste, Umami taste

\section{Background}

The role of sensory taste perception in childhood obesity Consumer studies have shown that sensory taste characteristics of foods are important drivers of food choice [1]. Different preferences may lead to distinctive food patterns that in turn may be related to diet-related health outcomes. There is evidence that such food patterns develop early in childhood and adolescence and then carry on into adulthood [2,3]. Few studies on this topic have been conducted in children, and none has

Correspondence: ahrens@bips.uni-bremen.de

Leibniz Institute for Prevention Research and Epidemiology - BIPS, Achterstrasse 30, D-28359 Bremen, Germany employed an international, multicentre epidemiological design. The European epidemiological multicentre study IDEFICS that addressed dietary, lifestyle, social and environmental determinants of children's health created a novel framework for the assessment of sensory taste perceptions of pre-adolescent children. The population-based approach of the study allows the investigation of the determinants of taste perceptions and their association with health outcomes like obesity in childhood [4]. Its prospective design allows for the longitudinal investigation of health outcomes in relation to dietary patterns.

With regard to sensory taste perception, the following research questions were addressed: (1) To what degree 
does sensory taste perception vary in European children? (2) Are taste thresholds or taste preferences associated with food choice or health outcomes? (3) Does new knowledge on sensory taste perception offer new opportunities for primary prevention of diet-related disorders? The cross-sectional analysis of the study shows substantial variation of objectively measured taste preferences and sensitivity across different European countries, indicating a likely effect of different food cultures on the sensory taste perception of children. An increased preference for fat and sugar seems to be associated with overweight and obesity, particularly in girls. Correspondingly, the longitudinal analysis revealed an increased risk for an elevated weight gain in children having a dietary pattern characterised by sweet and fatty foods while this risk was reduced in children with a pattern favouring fruits, vegetables and wholemeal bread. As it seems that dietary preferences are modifiable, preventive efforts may aim at shaping these preferences in a favourable direction already early in childhood.

\section{Methodological approach}

The IDEFICS (Identification and prevention of Dietary and lifestyle-induced health EFfects In Children and infantS) study is a multilevel epidemiological study using a European multicentre approach. The study started with a baseline survey of more than 16,000 children who were 2 to 9 years old. It has two main aims, with a strong focus on overweight and obesity in children: (1) To investigate the complex interplay of aetiological factors associated with diet- and lifestyle-related diseases and disorders in a population-based sample of children by means of cross-sectional and longitudinal analyses. A highly standardised protocol was implemented to assess the prevalence of overweight and obesity, related comorbid conditions and major risk factors. Objective measurements of weight status and related health outcomes such as blood pressure, insulin resistance and behavioural determinants such as physical activity are complemented by parent-reported data on diet, social/psychological factors and consumer behaviour. These standardised data allow the comparison of the prevalence and trajectory of health outcomes like childhood obesity and a multitude of risk factors and covariates across a diverse range of European cultures, climate zones and environments represented by eight countries [4-6]. (2) To complement the aetiological approach of the IDEFICS study by a community-oriented intervention programme for primary prevention of obesity in a controlled study design. Here, the study examines the effectiveness of a coherent set of intervention messages to improve diet and physical activity as well as to strengthen coping with stress [7]. The weight status of children was classified according to the age- and sex-specific reference curves of the International Obesity Task Force [8].
We aimed to identify factors associated with taste preference and taste sensitivity. Since sensory testing of free-living children has rarely been done outside the laboratory setting before and because the multicentre design of the study called for a simple and robust method that is not vulnerable to an observer bias, a new method had to be developed and tested for its feasibility and reliability. Based on existing norms like the DIN (German Institute for Standardisation) and long-standing experience with the sensory testing of new food products, a test system was developed under the lead of the Department of Food Technology and Bioprocess Engineering of the Technologie-Transfer-Zentrum Bremerhaven (TTZ). Procedures, substrates and concentrations were tested and adapted in an iterative process with 191 randomly selected boys and girls aged 4 to 7 years from kindergartens and primary schools [9]. It turned out that the taste thresholds of small children are up to an order of magnitude above those of adults. Concentrations of test solutions had to be adapted accordingly.

Since it became obvious that pre-school children wanted to please the examiner by reacting as supposedly desired, the final test protocol was worked out for primary school children aged 6 to 10 years and examiners were trained in avoiding suggestive phrasing of questions or gestures. For optimal standardisation, all stock solutions for the threshold test as well as the juices and test crackers for the preference tests were produced centrally and then shipped to all study locations. A standard operating procedure (SOP) was worked out to ensure standardisation of all tests across study centres and field staff and to minimise measurement bias. Besides the central training of the field staff, the SOP included the following requirements: examiners were advised not to smoke at least $1 \mathrm{~h}$ before the test, not to drink coffee or alcohol, not to eat peppermint or strong bubblegum and not to use too much perfume (preferably no perfume at all). Parents had to make sure that the children did not eat or drink (except water) for at least $1 \mathrm{~h}$ and that they did not chew peppermint or bubblegum. All materials had to be cleaned with neutral washing liquids free of perfumes.

A random subsample of 1,839 (20.8\%) IDEFICS schoolchildren aged 6 to 9 years from Italy, Estonia, Cyprus, Belgium, Sweden, Germany, Hungary and Spain agreed to participate in the sensory taste preference and taste sensitivity tests; 1,705 of them actually provided complete preference data. Tests were usually performed in the morning at the premises of the schools that the children attended.

For the assessment of taste sensitivity, a paired comparison staircase method, i.e. a threshold test, was arranged as a cardboard game where a range of five test solutions were ordered by concentration for each basic taste, i.e. sweet, salty, bitter and umami (in this order). Concentration 
ranges were as follows: sucrose $8.8-46.7 \mathrm{mmol}^{-1}$, sodium chloride $3.4-27.4 \mathrm{mmol}^{-1}$, caffeine $0.26-1.3 \mathrm{mmol}^{-1}$ and monosodium glutamate (MSG) $0.6-9.5 \mathrm{mmol}^{-1}$. The water-based solutions were offered in small cups (volume $20 \mathrm{ml}$ ). Children were asked to act as "taste detectives". They had to find out which of the cups contained pure water and which of them would taste different from pure water. Children were advised to compare each test solution against a reference cup containing distilled water and to put the respective cup on the appropriate field on the board (Figure 1). The lowest concentration at which the child claimed a difference to the reference sample was defined as the threshold concentration. Children were classified as sensitive for the respective taste if their threshold was below the median threshold concentration of the full sample.

The taste preference test was designed as a paired forced choice test using another cardboard (Figure 2). Elevated concentrations of sucrose and apple flavour in apple juice had to be compared with apple juice containing $0.53 \%$ added sucrose in a pairwise manner. The amount of sucrose was increased to $3.11 \%$ to assess the preference for sweet while $0.05 \%$ of commercially available apple flavour was added to assess flavour preference.

Increased levels of fat, sodium chloride and monosodium glutamate in crackers had to be compared against a standard reference cracker. Crackers were heartshaped and coated with $0.5 \%$ aqueous solution of soda lye to make them more attractive. To improve their texture, an emulsifying agent had to be added to the MSG- and salt-enriched crackers. The recipe and its variation for the cracker are summarised in Table 1 . The test sequence was as follows: (1) apple juice basic taste versus apple juice with added sugar, (2) apple juice basic taste versus apple juice with added apple flavour, (3) cracker basic recipe versus cracker with added fat, (4) cracker basic recipe versus cracker with added salt and (5) cracker basic recipe versus cracker with added MSG.

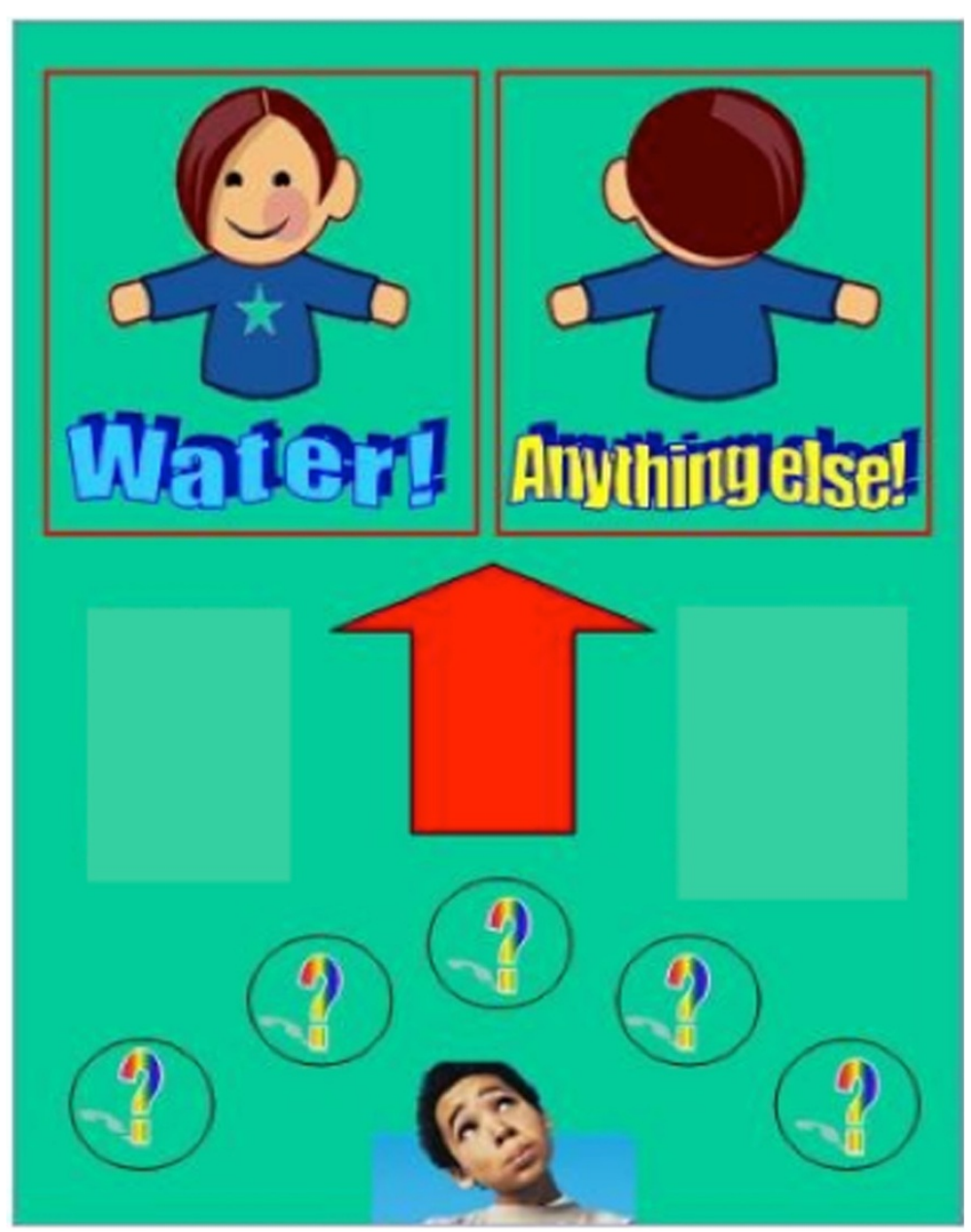

Figure 1 Board game for the taste threshold test. Children were advised to put the tested sample cup on the "water" field if they tasted no difference to the reference sample and on the other field if they indeed tasted a difference. 


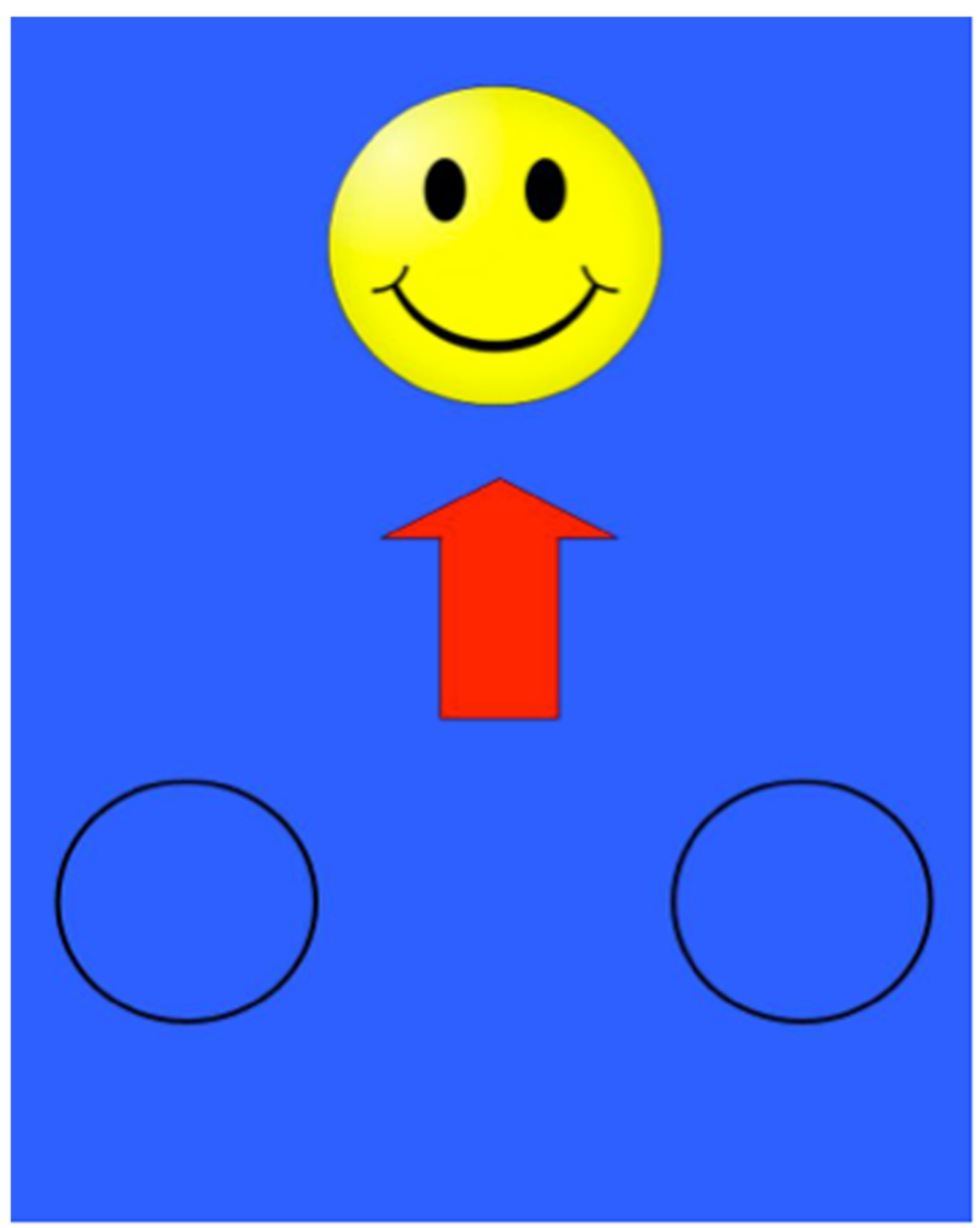

Figure 2 Cardboard used to test the taste preference. Children were advised to put the preferred taste on the smiley.

A parent or guardian living with the child filled in a proxy questionnaire to record age, sex, country of residence, parental education and feeding practices including breastfeeding, first introduction of fruit, TV exposure and using food as a reward or punishment. To report on the usual frequency of the consumption of selected food items and on dietary habits, parents completed the Children's Eating Habits Questionnaire [10,11]. The latter provided the basis for the identification of the actual dietary patterns by principal component analysis [12].

The statistical analysis included chi-square tests to assess differences by survey centre. Odds ratios and their
95\% confidence intervals were calculated by a logistic regression analysis to identify predictors and correlates of a preference for sweet, fat, salty and umami taste. Age, sex, parental education, survey centre, breastfeeding and age at introduction of fruits were included in the statistical model as possible causal predictors of taste preferences. TV use, using food as a reward and taste sensitivity were considered as correlates because the direction of an association with taste preferences would not be clear in a cross-sectional analysis like ours. For example, if taste sensitivity is modifiable by environmental factors or dietary behaviour rather than being a stable, genetically

Table 1 Recipe of the cracker to determine fat, salt and umami preference

\begin{tabular}{lccccc}
\hline Type of cracker & Flour/water (\%) & Salt (\%) & Fat (\%) & MSG (\%) & DAWE (\%) \\
\hline Reference & 91.3 & 0.7 & 8 & 8 & 0 \\
Salt & 89.4 & 1.6 & 18 & 0 & 1 \\
Fat & 81.3 & 0.7 & 8 & 1 & 0 \\
Umami & 89.3 & 0.7 & 8 & 1 \\
\hline
\end{tabular}

DAWE diacetyl tartaric ester (emulsifying agent), MSG monosodium glutamate. 
determined trait, then it may well be that preferences influence preferences and vice versa. Additional analyses were stratified by survey centre where odds ratios were only adjusted for age, sex and parental education. To account for multiple testing, a Bonferroni adjustment of the significance level was done.

\section{Statement of Ethics}

We certify that all applicable institutional and governmental regulations concerning the ethical use of human volunteers were followed during this research. Approval by the appropriate Ethics Committees was obtained by each of the 8 centres doing the fieldwork. Study children did not undergo any procedures unless both they and their parents had given consent for examinations, collection of samples, subsequent analysis and storage of personal data and collected samples. Study subjects and their parents could consent to single components of the study while abstaining from others.

\section{Findings \\ Prevalence of sensory taste sensitivity and sensory preferences}

The prevalence of taste sensitivity differs substantially between countries for each of the four basic tastes. The sensitivity for all tastes tends to be generally below average among children from Cyprus. The highest prevalence values were observed for sweet sensitivity in Italian and Estonian children, for bitter sensitivity in Hungarian and Spanish children and for umami in Hungarian children. The prevalence of salt sensitivity varied less between most countries; only in children from Cyprus and Belgium the corresponding prevalence was clearly below the average (Figure 3).

Regarding sensory preferences, most children preferred the food sample with the added flavouring substance for sweet, fat and salt (Figure 4). However, only $34 \%$ of the children preferred the cracker with added MSG on the natural cracker. The preference for the added ingredient tends to be generally higher in Hungarian, Spanish and Estonian children. The preference prevalence varies substantially between countries, particularly for fat and umami. The preference prevalence for umami is more than twofold higher in Estonia and Spain as compared to Cyprus and Belgium while the preference for fat is almost twice as high in Estonia and Germany as compared to Cyprus. The preference for the salty cracker is highest in Estonia and lowest in Cyprus and Italy. Sweet preference shows the smallest variation by country, with the lowest prevalence values in Germany and Cyprus. Taste preferences were not significantly associated with each other with the exception of fat and umami. Children preferring the fat-added cracker also had a tendency to prefer the

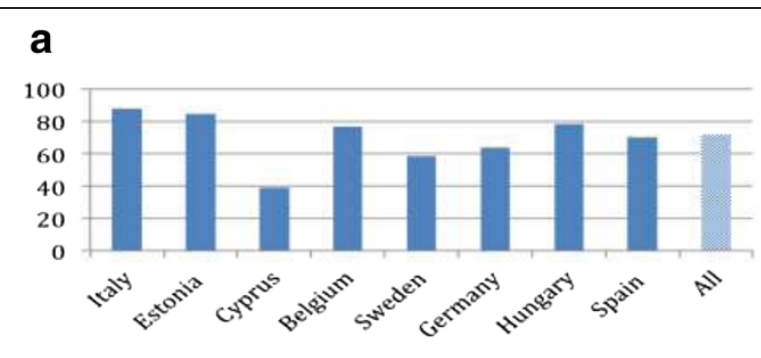

\section{b}

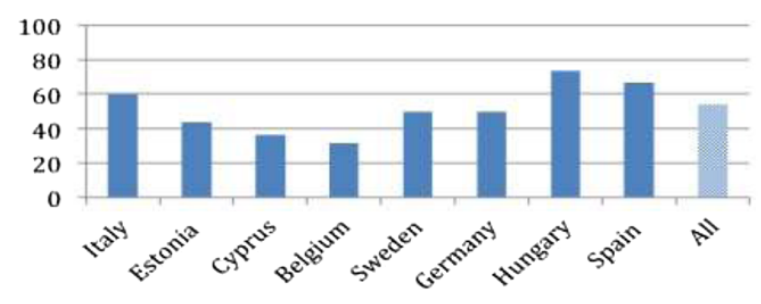

C

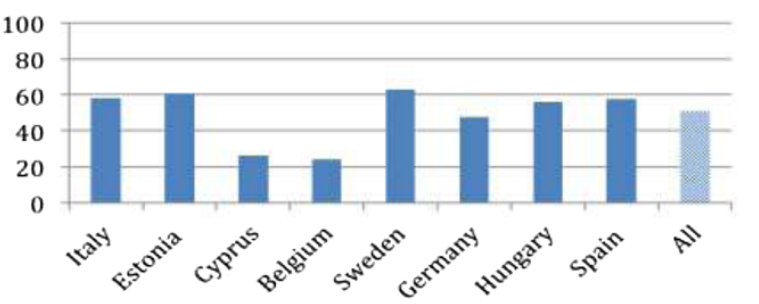

d

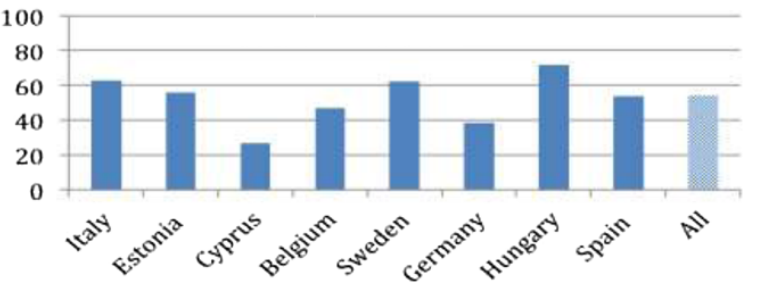

Figure 3 Proportion of children sensitive to four basic taste qualities by country. (a) Sweet sensitive, (b) bitter sensitive, (c) salt sensitive and (d) umami sensitive.

sugar-added apple juice, but this association was only weak and statistically non-significant.

\section{Correlates and consequences of sensory taste preferences} Country of residence is the strongest factor related to preferences for all four taste qualities. No sex differences are observed for any of the taste qualities, but taste preferences differ by age. While the preference for sugaradded juice seems to increase by age, the fat-added cracker is less preferred in 8- to 9-year-olds as compared to 6-year-old children. Also, the preference for salt increases with age while it decreases for MSG. Parental education, early feeding habits, TV viewing, using food as a reward and taste thresholds were not consistently related to taste preferences [13]. 


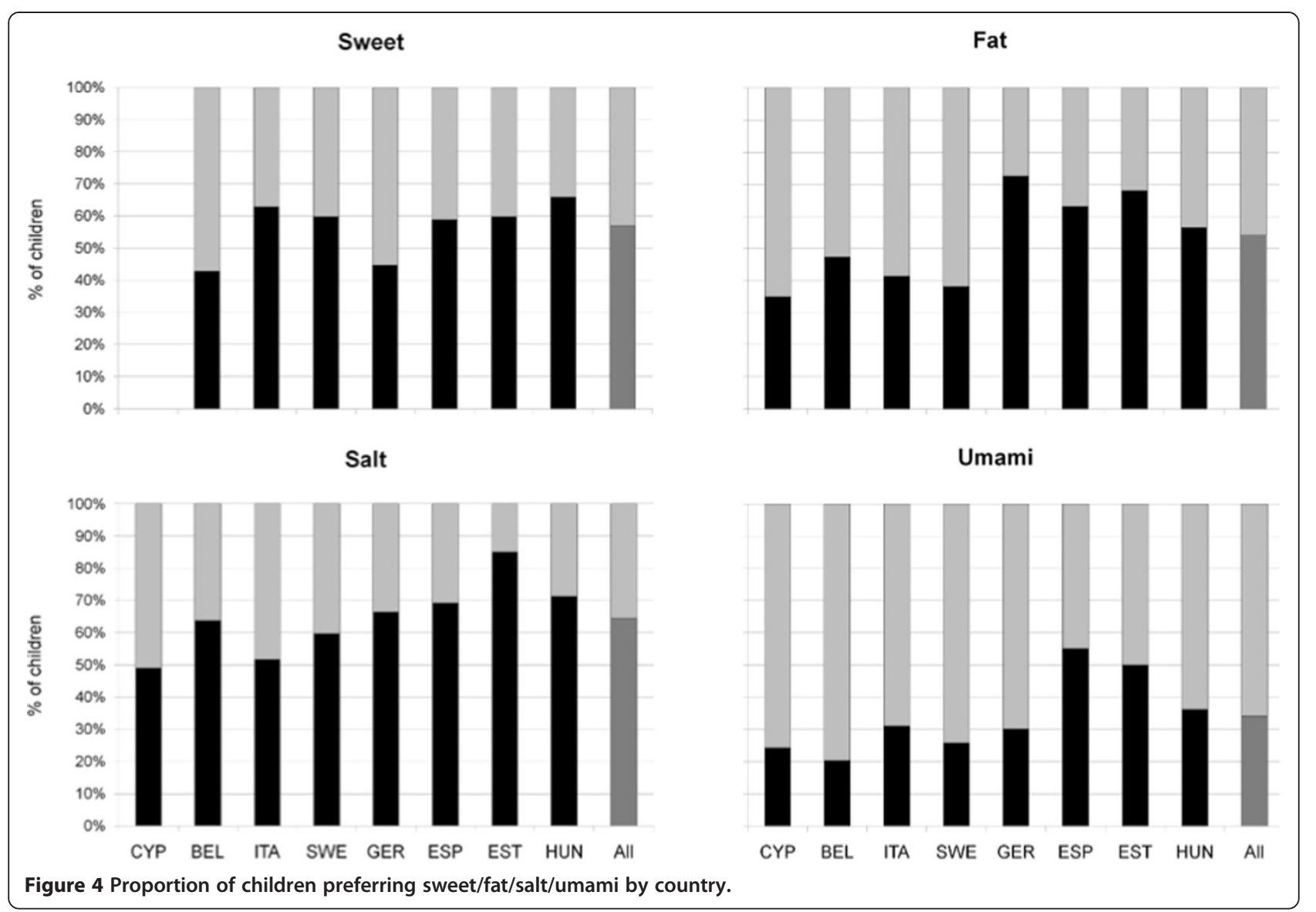

We also investigated the association between taste preferences and dietary patterns. Children's consumption frequency of fatty and sweet foods was obtained from the food frequency questionnaire (FFQ) completed by a parent for his/her child. Frequent consumption of fatty foods shows an association with fat preference in bivariate analyses, but adjustment for country attenuates this association. No such association is observed for sweet preference and the parent-reported consumption of sweet foods, neither in crude nor in adjusted analyses [14]. Although the reliability of the FFQ was reasonably good [11], the absence of strong associations between objectively measured taste preferences and parent-reported food consumption frequencies may be explained by misclassification of proxy-reported food consumption as indicated by the non-negligible degree of within-subject variation between repeated reports [11].

Weight and height of the children were measured according to highly standardised procedures. Regardless of the country of residence, age, sex, parental education and parental BMI, overweight and obesity were positively associated with preference for fat-enriched crackers and with sugar-sweetened apple juice. The odds of being overweight or obese are elevated by $50 \%$ among children preferring the fat-added cracker as compared to children preferring the natural cracker (Figure 5). Children preferring the sugar-sweetened juice also show $50 \%$ higher odds of being overweight or obese as compared to children preferring the natural juice (Figure 5). Fat preference associations were stronger in girls. Girls but not boys who simultaneously preferred fatty crackers and sweetened juice reveal a particularly high probability of being overweight or obese [14]. Preference for salt, MSG or apple flavour does not seem to be associated with weight status.

Although the direct association between taste preferences and reported frequency of corresponding food items was relatively weak, we hypothesise that the observed positive association between sensory fat and sweet preference and weight status in our children may be mediated through a corresponding food choice pattern. This hypothesis is supported by the analysis of observed dietary patterns in relation to weight gain. Using a principal component analysis, we were able to identify four distinct dietary patterns [12]: (1) "Snacking" is characterised by the consumption of sandwiches (including hamburgers, hotdogs and kebabs); butter or margarine on bread; snacks, savoury pastries, fritters; snacks, chocolate, candy bars; and white bread, white 


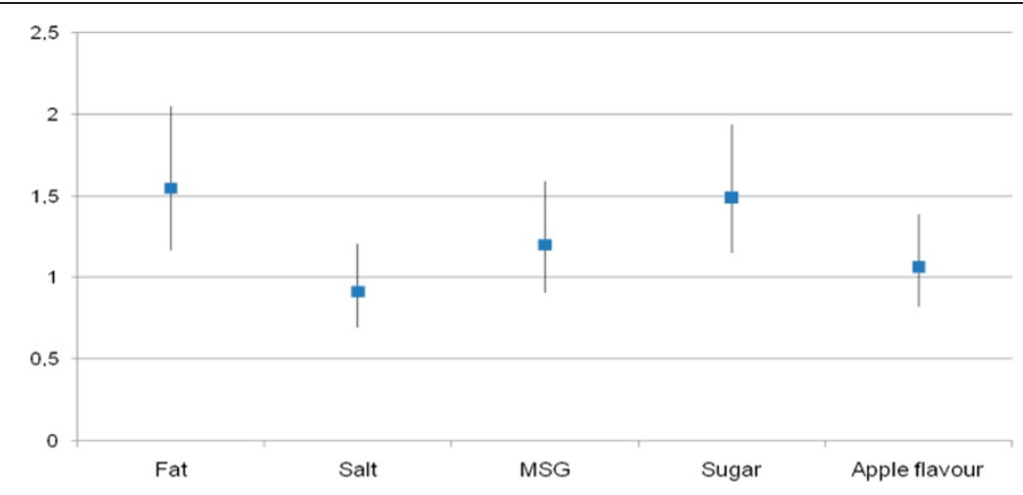

Figure 5 Odds ratios and $95 \%$ confidence intervals adjusted for age, sex and country for overweight/obesity in children with preference for added fat, salt and MSG in crackers and for added sugar and apple flavour in apple juice. Natural cracker and natural apple juice served as the reference categories, respectively.

rolls, crispbread. (2) "Sweet and fat" is characterised by the consumption of chocolate- or nut-based spreads; biscuit cakes, pastries and puddings; sweets/candy; fried meats; and soft drinks. (3) "Vegetables and wholemeal" is characterised by the consumption of raw vegetables; wholemeal bread; cooked vegetables; fresh fruit without added sugar; plain milk (not sweetened); and porridge, muesli (not sweetened). (4) "Proteins and water" is characterised by the consumption of fresh fish (not fried); water; fried fish, fish fingers; eggs (fried, scrambled), fresh meat (not fried); and pasta, noodles, rice. During a 2-year follow-up, those children adhering to the "sweet and fat" pattern (upper tertile) had a $17 \%$ increased risk for an excessive weight gain while this risk was reduced by $12 \%$ in children following the "vegetable and wholemeal" pattern (upper tertile) (Figure 6).

In another approach, we calculated the propensity of children to favourably consume sweet or fatty foods in order to investigate the association between overweight,
TV consumption and the adherence to an unhealthy food pattern [15]: The weekly consumption frequencies of each of 17 foods and beverages that are high in fat and of 12 foods and beverages with high sugar content were calculated for each of these categories. The other 14 items of the FFQ were also converted into weekly frequency scores. A continuous propensity score was calculated by dividing the total weekly frequency for the high-sugar or high-fat items by the individual's total consumed food frequencies. These propensity scores were meant to reflect the proportions of sugary and fatty foods in the whole diet of a child. Dietary fat propensity was calculated as the ratio of fried potatoes, whole fat milk, whole fat yogurt, fried fish, cold cuts/sausages, fried meat, fried eggs, mayonnaise, cheese, chocolate- or nut-based spread, butter/margarine on bread, nuts/seeds/dried fruit, salty snacks, savoury pastries, chocolate-based candies, cake/ pudding/cookies and ice cream to total frequencies/week. Sugar propensity was calculated as the ratio of fruit with

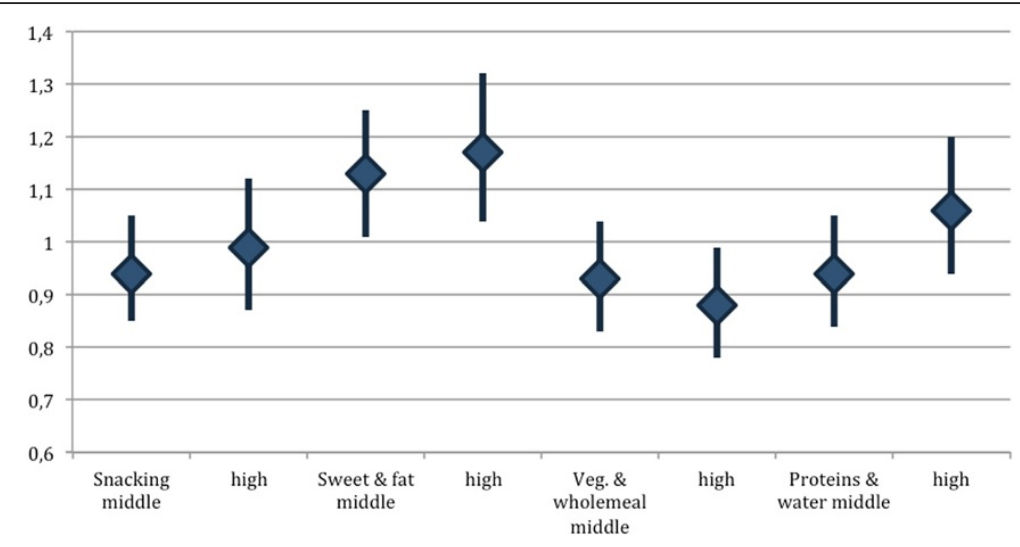

Figure 6 Risk of increased BMI $z$-score (+20\%) over 2 years of follow-up by food pattern. Odd ratios (OR) with $95 \%$ confidence intervals from mixed effects logistic regression with country as "random effect", adjusted for sex, age, hours of physical activity/week (continuous), country specific income (low, low/medium, medium, medium/high and high). The lowest tertile of each pattern was used as the reference category; middle $=$ second tertile and high $=$ upper tertile. 
added sugar, fruit juice, sugar-sweetened drinks, sweetened breakfast cereals, sweetened milk, sweetened yogurt, jam/ honey, chocolate- or nut-based spread, chocolate-based candies, non-fat candies, cake/pudding/cookies and ice cream to total frequencies/week. These two propensity scores were divided into quartiles to assess their association with children's TV consumption using odds ratios. This analysis shows that the propensity of children to consume foods high in fat or sugar is positively and steadily associated with indicators of frequent TV consumption (Figure 7). At the same time, these indicators are associated with a $20 \%$ to $30 \%$ increased risk for being overweight or obese [15]. We may speculate that a higher exposure to TV programmes-and consequently to food advertisements that mostly promote unhealthy foods-could influence dietary patterns of children in an unfavourable direction. The observed association of high TV consumption with, both, overweight and an unfavourable propensity to consume sugary and fatty foods may indeed provide a starting point for the primary prevention of childhood overweight.

\section{Conclusion}

We conclude that culture and age may be important determinants of taste preferences in children younger than 10 years of age. Fat and sweet taste preferences show a positive association with weight status in European children across regions with varying food cultures. The propensity to consume foods with a high content of fat and sugar is associated with indicators of high TV consumption that in turn is more prevalent in overweight and obese children. These associations are based on a crosssectional analysis, and conclusions about causality of the

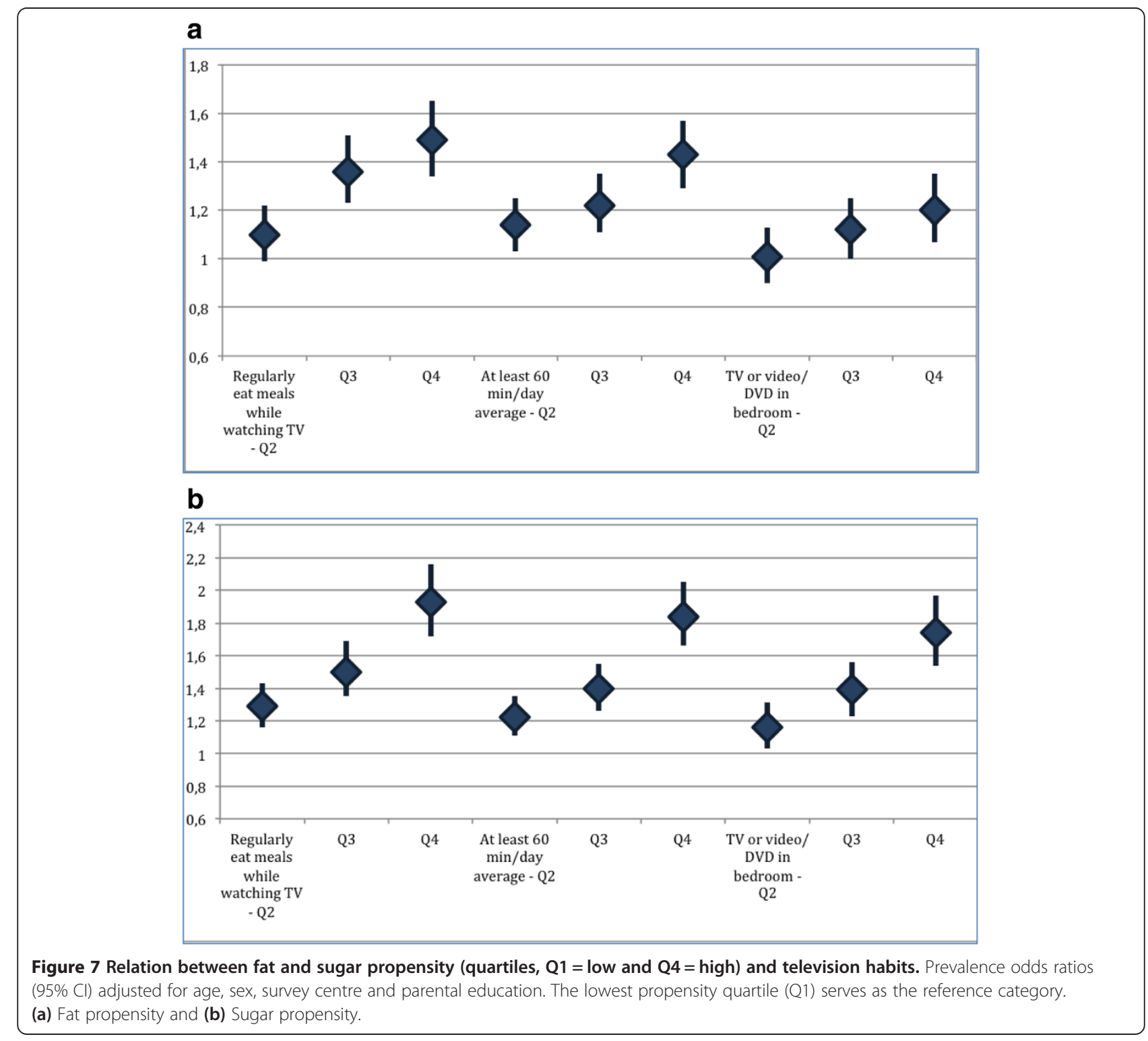


associations should thus be drawn with great caution. Nevertheless, the data presented are in agreement with the hypothesis that preference for sweet and fatty foods parallels a higher propensity to consume these foods. The positive longitudinal association of an unhealthy food pattern characterised by sweet and fatty foods with an unfavourable weight trajectory in children provides evidence for a causal relationship. Thus, it seems plausible that food preferences of children are shaped by cultural, behavioural and environmental factors including exposure to TV and other media. Ultimately, unfavourable preferences may result in less favourable food patterns which then lead to negative health outcomes like obesity.

\section{Abbreviations \\ DAWE: diacetyl tartaric ester; FFQ: food frequency questionnaire; MSG: monosodium glutamate; TTZ: Technologie-Transfer-Zentrum} Bremerhaven.

\section{Competing interests}

The author declares that he has no competing interests.

\section{Authors' information}

Prof. Dr. Wolfgang Ahrens is a professor of epidemiological methods at the University of Bremen and the Deputy Director of the Leibniz Institute for Prevention Research and Epidemiology where he leads the Department of Epidemiological Methods and Etiologic Research. His current research focuses on the causes of chronic diseases as well as their primary prevention. He coordinates the largest Europe-wide cohort study on overweight, obesity and related disorders in children focusing on nutrition, lifestyle and social factors (www.ideficsstudy.eu; www.ifamilystudy.eu), and he is one of the scientific directors of the National Cohort in Germany (www.nationale-kohorte.de).

\section{Acknowledgements}

This work was done as part of the IDEFICS study (www.idefics.eu). We gratefully acknowledge the financial support of the European Community within the Sixth RTD Framework Programme Contract No. 016181 (FOOD). We are grateful to the participating children and their parents for participating in the study, and we thank the field teams for collecting the data.

Received: 2 December 2014 Accepted: 2 December 2014

Published: 4 March 2015

\section{References}

1. Glanz K, Basil M, Maibach E, Goldberg J, Snyder D: Why Americans eat what they do: taste. J Am Diet Assoc 1998, 98(10):1118-1126.

2. Tabacchi G, Giammanco S, La Guardia M, Giammanco M: A review of the literature and a new classification of the early determinants of childhood obesity: from pregnancy to the first years of life. Nutr Res 2007, 27(10):587-604.

3. Kelder SH, Perry CL, Klepp Kl, Lytle LL: Longitudinal tracking of adolescent smoking, physical activity, and food choice behaviors. Am J Public Health 1994, 84(7):1121-1126.

4. Ahrens W, Bammann K, Siani A, Buchecker K, De Henauw S, lacoviello L, Hebestreit A, Krogh V, Lissner L, Mårild S, Molnár D, Moreno LA, Pitsiladis YP, Reisch L, Tornaritis M, Veidebaum T, Pigeot I: The IDEFICS cohort: Design, characteristics and participation in the baseline survey. Int J Obes 2011, 35(Suppl 1):3-15.

5. Ahrens W, Bammann K, De Henauw S, Halford J, Palou A, Pigeot I, Siani A, Sjöström M, European Consortium of the IDEFICS Project: Understanding and preventing childhood obesity and related disorders - IDEFICS: a European multilevel epidemiological approach. Nutr Metab Cardiovasc Dis 2006, 16:302-308.

6. Bammann K, Peplies J, Sjöström M, Lissner L, De Henauw S, Galli C, Lacoviello L, Krogh V, Mårild S, Pigeot I, Pitsiladis Y, Pohlabeln H, Reisch L,
Siani A, Ahrens W: Assessment of diet, physical activity biological, social and environmental factors in a multi-centre European project on diet and lifestyle-related disorders in children (IDEFICS). J Public Health 2006, 14:279-289.

7. De Henauw S, Verbestel V, Marild S, Barba G, Bammann K, Eiben G, Hebestreit A, lacoviello L, Gallois K, Konstabel K, Kovács E, Lissner L, Maes L, Molnár D, Moreno LA, Reisch L, Siani A, Tornaritis M, Williams G, Ahrens W, De Bourdeaudhuij I, Pigeot I: The IDEFICS community-oriented intervention program: a new model for childhood obesity prevention in Europe? Int $\rfloor$ Obesity 2011, 35(Suppl 1):16-23.

8. Cole TJ, Bellizzi MC, Flegal KM, Dietz WH: Establishing a standard definition for child overweight and obesity worldwide: international survey. BMJ 2000, 320:1240-1243.

9. Knof K, Lanfer A, Bildstein MO, Buchecker K, Hilz H, IDEFICS Consortium: Development of a method to measure sensory perception in children at the European level. Int J Obes (Lond) 2011, 35(1):S131-S136.

10. Huybrechts I, Boernhorst C, Pala V, Moreno LA, Barba G, Lissner L, Fraterman A, Veidebaum T, Hebestreit A, Sieri S, Krogh V, Ottevaere C, Tornaritis M, Molnar D, Ahrens W, De Henauw S, on behalf of the IDEFICS consortium: Evaluation of the children's eating habits questionnaire used in the IDEFICS study by relating urinary calcium and potassium to milk consumption frequencies among European children. Int J Obes 2011, 35(Suppl 1):69-78.

11. Lanfer A, Hebestreit A, Ahrens W, Krogh V, Sieri S, Lissner L, Eiben G, Siani A, Huybrechts I, Loit HM, Papoutsou S, Kovács E, Pala V, IDEFICS Consortium: Reproducibility of food consumption frequencies derived from the children's eating habits questionnaire used in the IDEFICS study. Int $J$ Obes 2011, 35(Suppl. 1):61-68.

12. Pala V, Lissner L, Hebestreit A, Lanfer A, Sieri S, Siani A, Huybrechts I, Kambek L, Molnar D, Tornaritis M, Moreno L, Ahrens W, Krogh V: Dietary patterns and longitudinal change in body mass in European children: a follow-up study on the IDEFICS multicenter cohort. Eur J Clin Nutr 2013, 67(10):1042-1049.

13. Lanfer A, Bammann K, Knof K, Buchecker K, Russo P, Veidebaum T, Kourides Y, De Henauw S, Molnar D, Bel-Serrat S, Lissner L, Ahrens W: Predictors and correlates of taste preferences in European children: the IDEFICS study. Food Qual Prefer 2013, 27(2):128-136.

14. Lanfer A, Knof K, Barba G, Veidebaum T, Papoutsou S, de Henauw S, Soós T, Moreno LA, Ahrens W, Lissner L: Taste preferences in association with dietary habits and weight status in European children: results from the IDEFICS study. Int J Obes (Lond) 2012, 36(1):27-34.

15. Lissner L, Lanfer A, Gwozdz W, Olafsdottir S, Eiben G, Moreno LA, Santaliestra-Pasías AM, Kovács E, Barba G, Loit HM, Kourides Y, Pala V, Pohlabeln H, De Henauw S, Buchecker K, Ahrens W, Reisch L: Television habits in relation to overweight, diet and taste preferences in European children: the IDEFICS study. Eur J Epidemiol 2012, 27(9):705-715.

doi:10.1186/2044-7248-4-8

Cite this article as: Ahrens: Sensory taste preferences and taste sensitivity and the association of unhealthy food patterns with overweight and obesity in primary school children in Europe-a synthesis of data from the IDEFICS study. Flavour 2015 4:8.

\section{Submit your next manuscript to BioMed Central and take full advantage of:}

- Convenient online submission

- Thorough peer review

- No space constraints or color figure charges

- Immediate publication on acceptance

- Inclusion in PubMed, CAS, Scopus and Google Scholar

- Research which is freely available for redistribution 